\title{
PHYSICS OF NONLINEAR OPTICS
}


This page is intentionally left blank 


\section{PHYSICS OF NONLINEAR OPTICS}

\section{Guang S. He}

State University of New York at Buffalo, USA

Song H. Liu

South China Normal University, CHINA 
Published by

World Scientific Publishing Co. Pte. Ltd.

5 Toh Tuck Link, Singapore 596224

USA office: Suite 202, 1060 Main Street, River Edge, NJ 07661

UK office: 57 Shelton Street, Covent Garden, London WC2H 9HE

\author{
Library of Congress Cataloging-in-Publication Data \\ He, Guang-Sheng. \\ Physics of nonlinear optics / Guang S. He, Song H. Liu. \\ p. $\mathrm{cm}$. \\ Includes bibliographical references and index. \\ ISBN 9810233191 \\ 1. Nonlinear optics. I. Liu, Song H. II. Title. \\ QC446.2.H6 1999 \\ $535^{\prime} \cdot 2-\mathrm{dc} 21$ \\ 99-39800 \\ CIP \\ British Library Cataloguing-in-Publication Data \\ A catalogue record for this book is available from the British Library.
}

First published 1999

Reprinted 2003

Copyright $\odot 1999$ by World Scientific Publishing Co. Pte. Ltd.

All rights reserved. This book, or parts thereof, may not be reproduced in any form or by any means, electronic or mechanical, including photocopying, recording or any information storage and retrieval system now known or to be invented, without written permission from the Publisher.

For photocopying of material in this volume, please pay a copying fee through the Copyright Clearance Center, Inc., 222 Rosewood Drive, Danvers, MA 01923, USA. In this case permission to photocopy is not required from the publisher. 
(a)

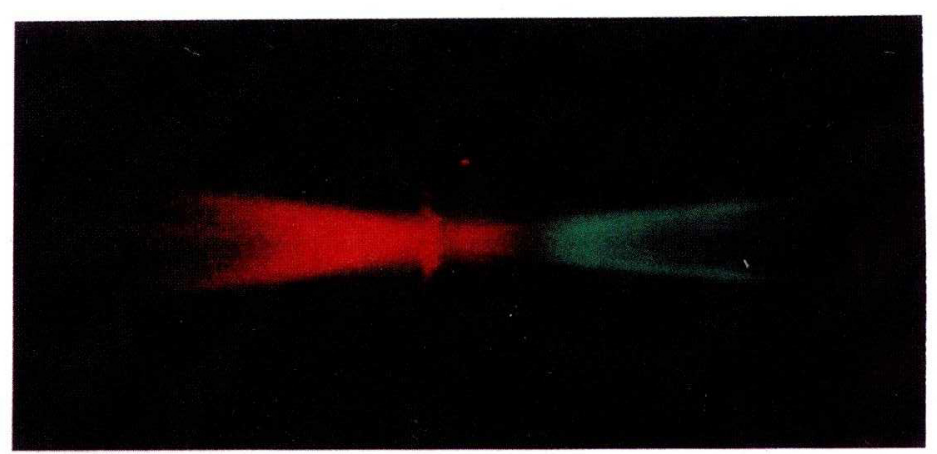

(b)

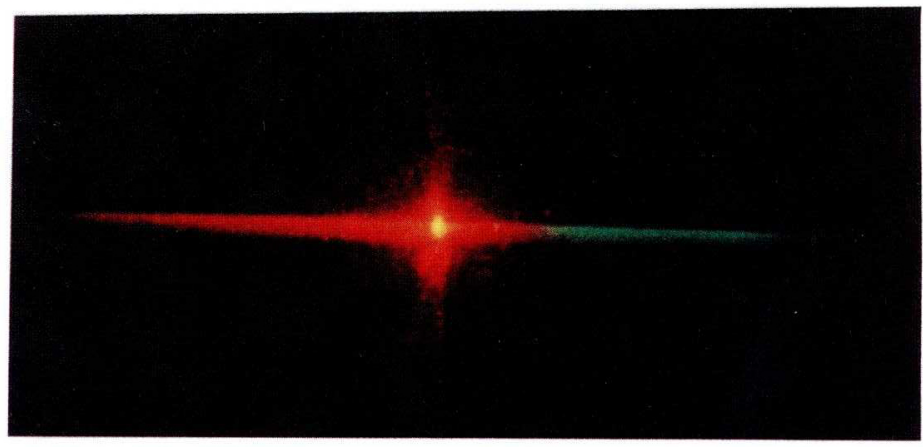

(c)

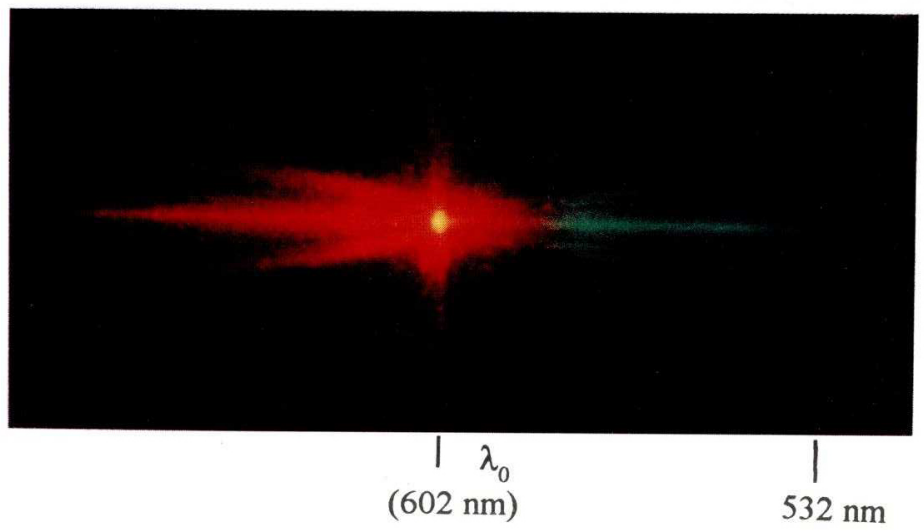

Photo 1 Angle-dependent spectral structure of continuum generation in heavy water $\left(\mathrm{D}_{2} 0\right)$ pumped with 602-nm, 0.5-ps laser pulses: (a) noncoUinear emission, (b) collinear emission, and (c) overall emission. (See the explanation for Fig. 7.11 in the text.) 
(a)

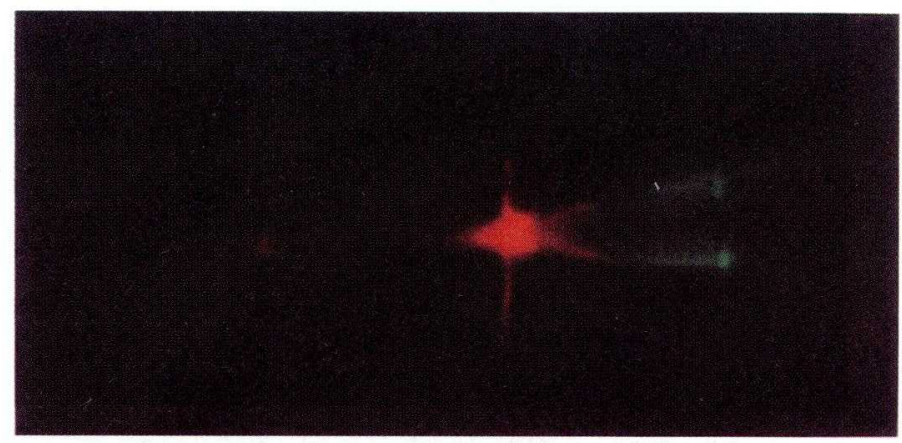

(b)

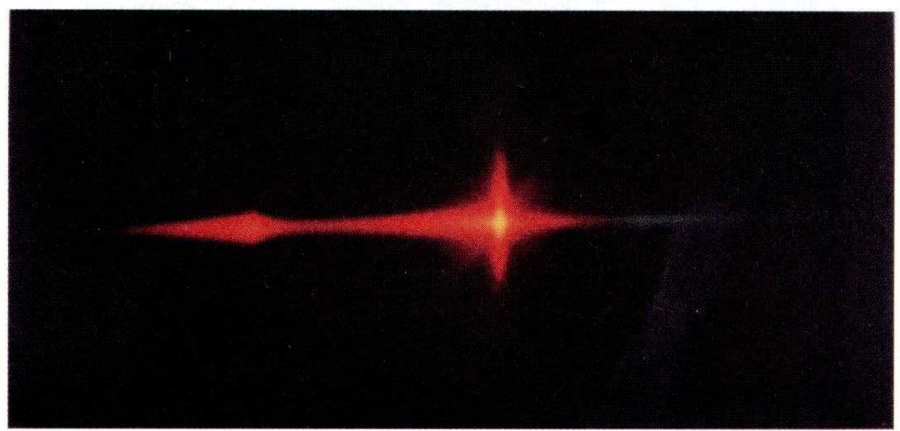

(c)

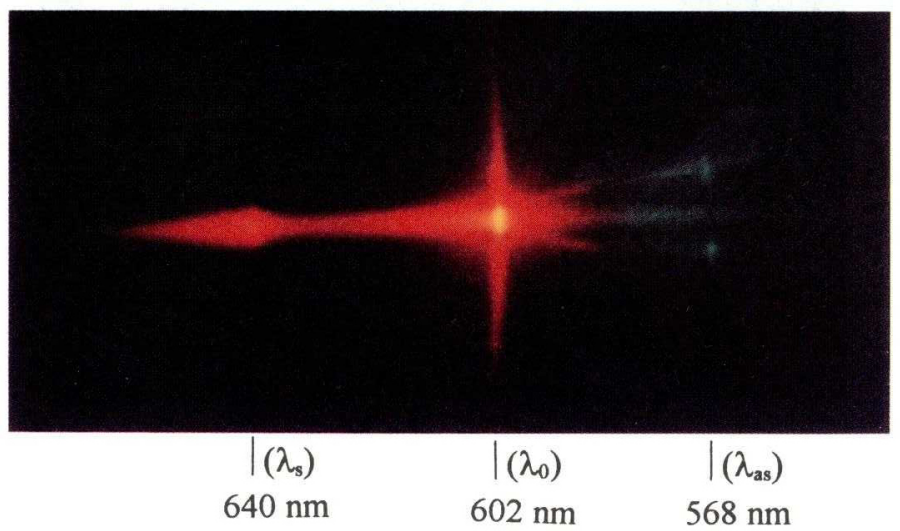

Photo 2 Spectral structure of coherent emission in benzene $\left(\mathrm{C}_{6} \mathrm{H}_{6}\right)$ pumped with 602-nm, 0.5-ps laser pulses: (a) noncollinear emission, (b) collinear emission, and (c) overall emission. (See the explanation for Fig. 7.12 in the text.) 
(a)

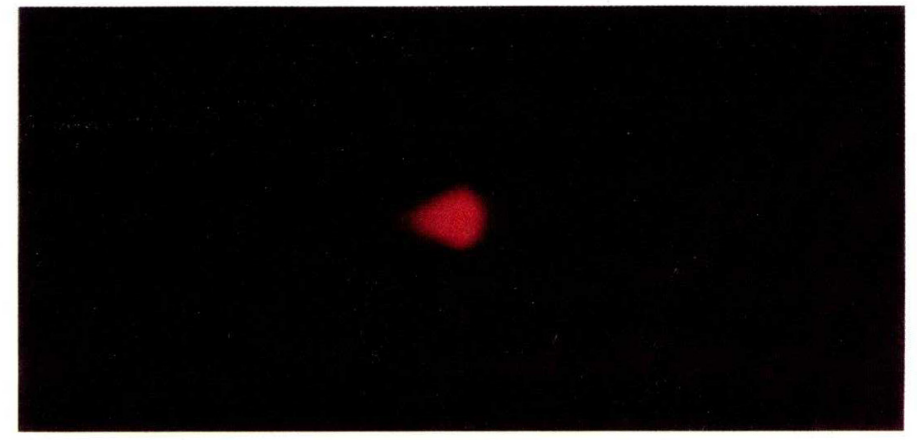

(b)

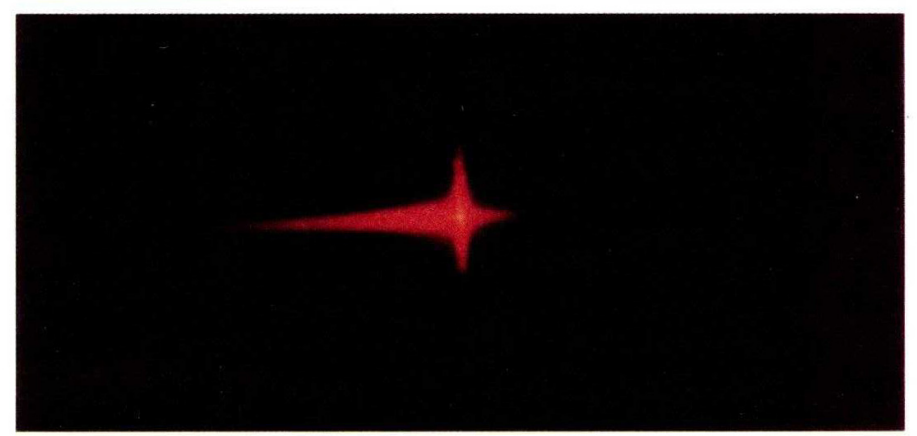

(c)

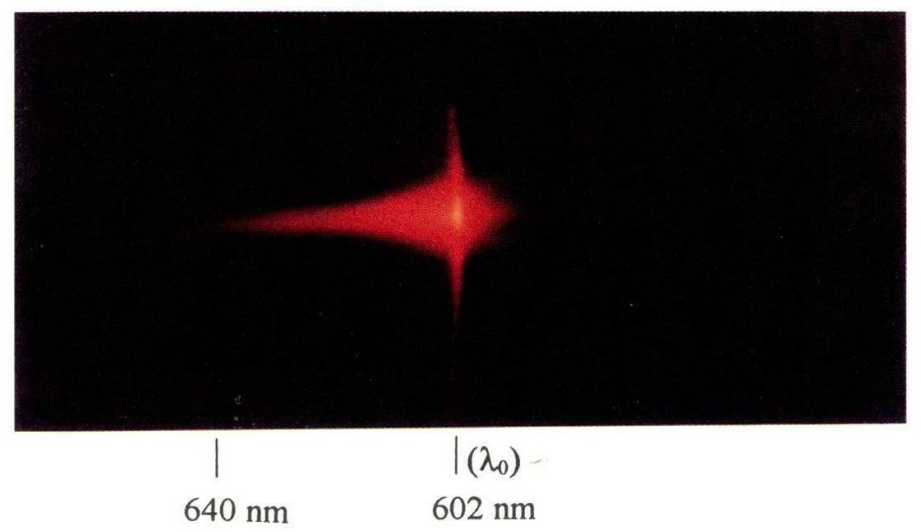

Photo 3 Spectral structure of coherent emission in carbon disulfide $\left(\mathrm{CS}_{2}\right)$ pumped with 602-nm, 0.5-ps laser pulses: (a) noncollinear emission, (b) collinear emission, and (c) overall emission. (See the explanation for Fig. 7.13 in the text.) 
(a)

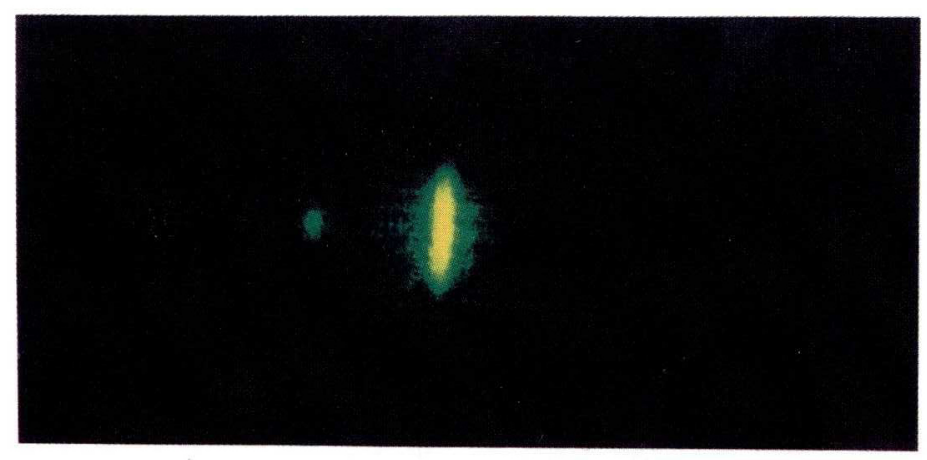

(b)

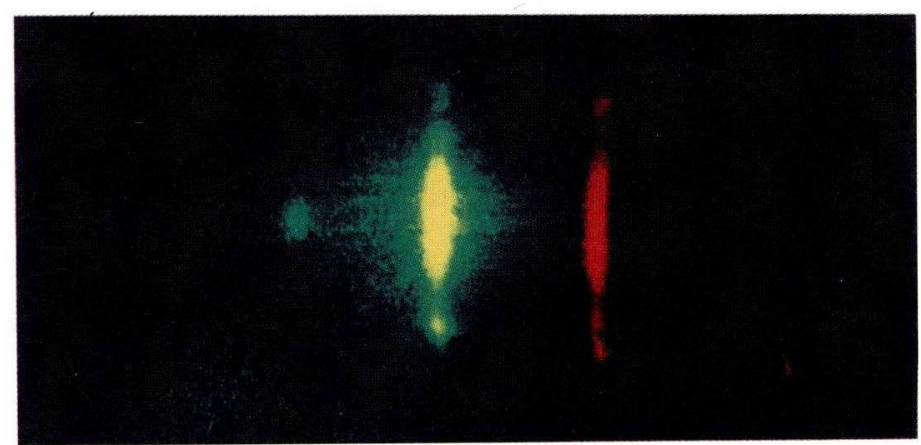

(c)

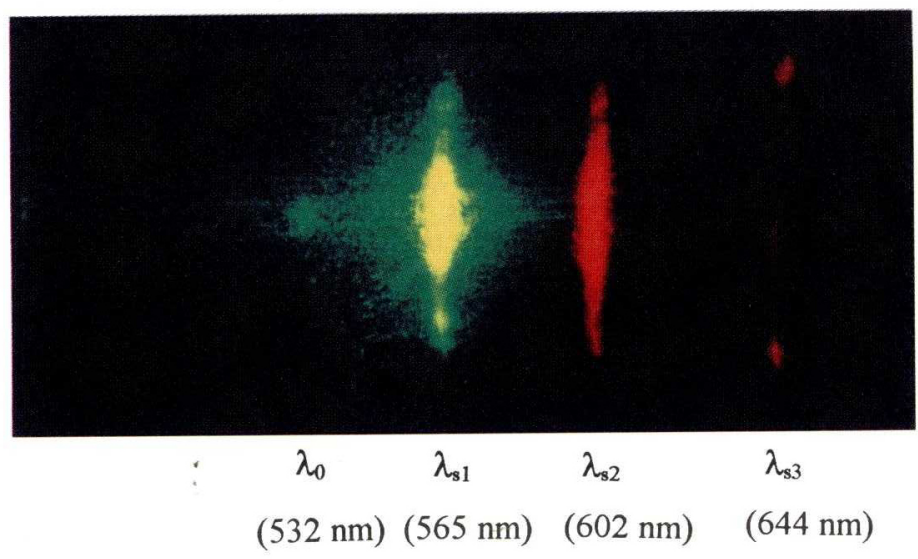

Photo 4 Spatial structures of Stokes spectral components of stimulated Raman scattering and four-wave-frequency-mixing in calcite crystal pumped with 532-nm, 8-ns laser pulses at three different intensity levels. (See the explanation for Fig. 8.7 in the text.) 
(a)

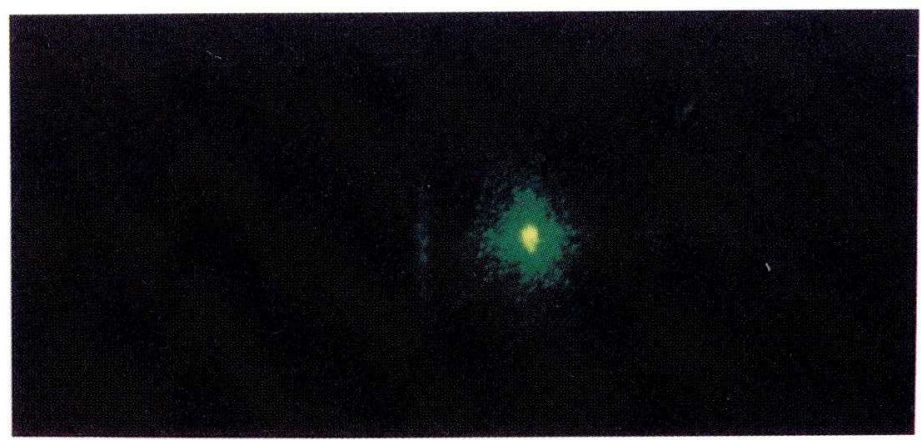

(b)

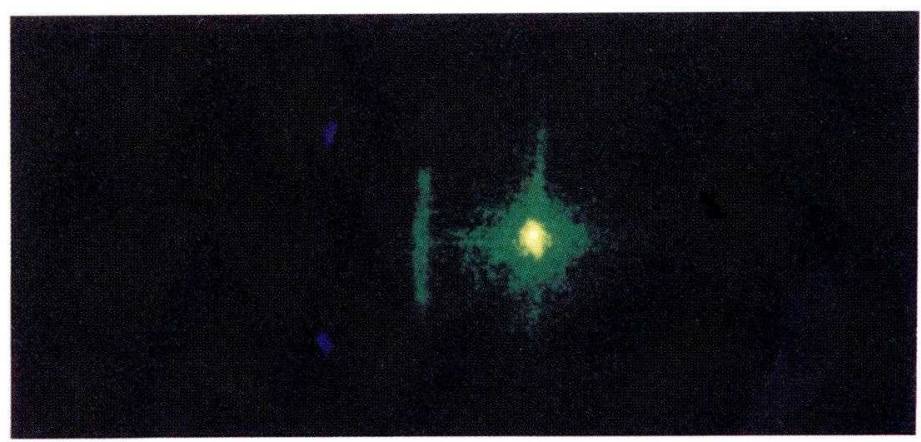

(c)

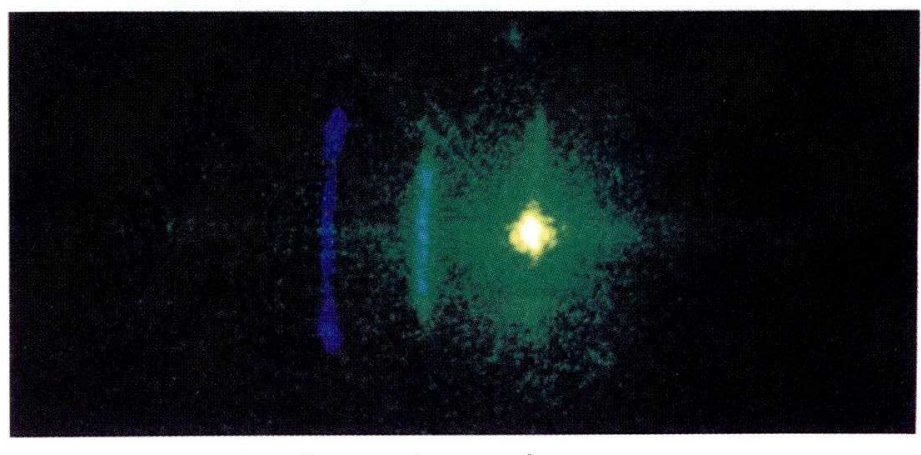

$(477 \mathrm{~nm})(503 \mathrm{~nm})(532 \mathrm{~nm})$

Photo 5 Spatial structures of anti-Stokes spectral components of stimulated Raman scattering and four-wave-frequency-mixing in calcite crystal pumped with 532-nm, 8-ns laser pulses at three different intensity levels. (See the explanation for Fig. 8.8 in the text.) 
(a)

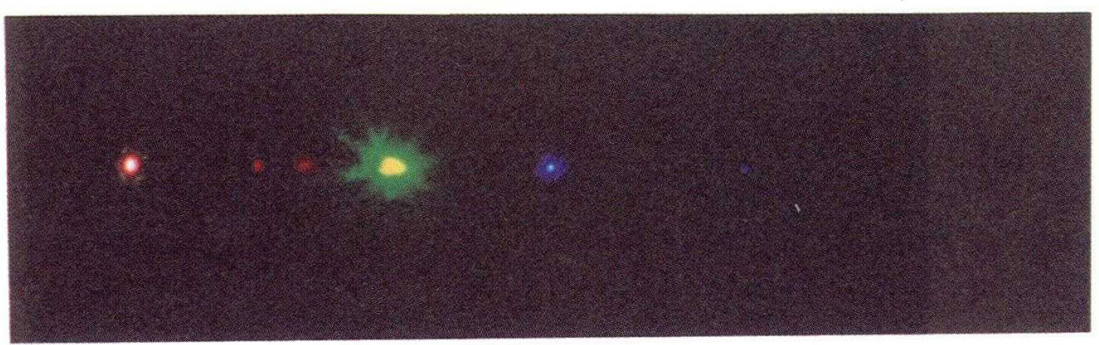

(b)

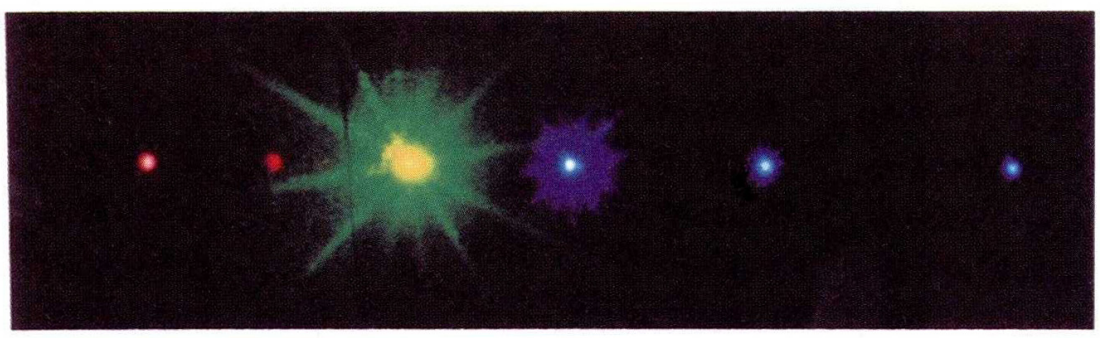

(c)

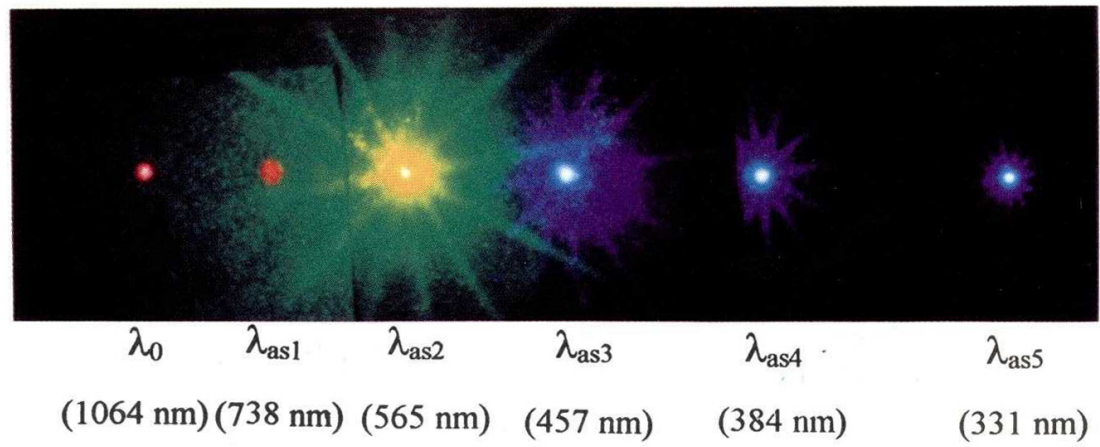

Photo 6 Spectra of multi-order anti-Stokes stimulated Raman scattering in highpressure hydrogen $\left(\mathrm{H}_{2}\right)$ sample pumped with 1064-nm, 8-ns laser pulses at three different intensity levels. (See the explanation for Fig. 8.10 in the text.) 
(a)

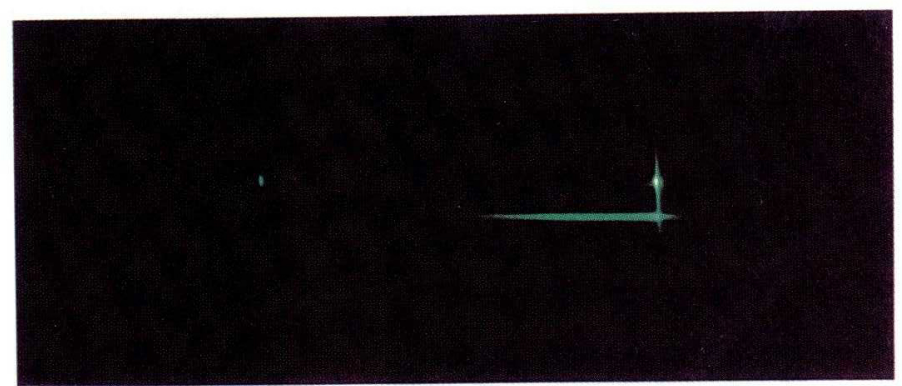

(b)

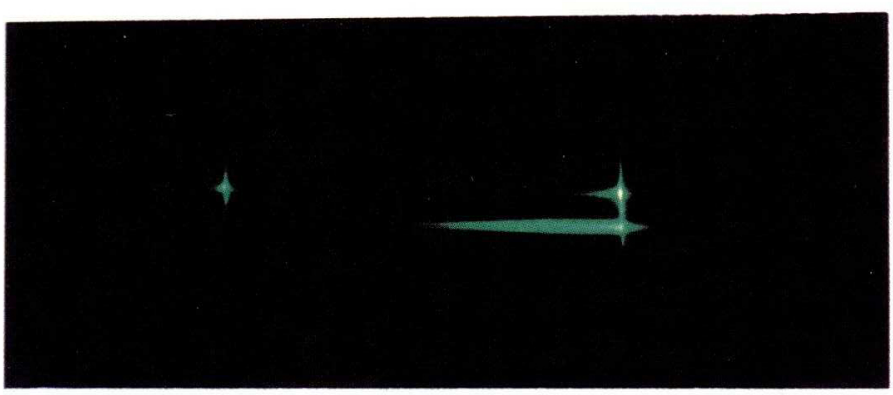

(c)

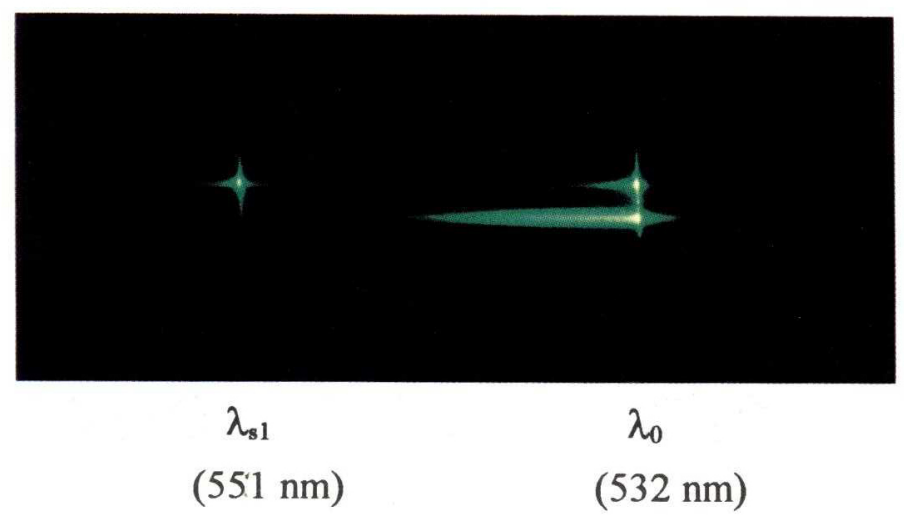

Photo 7 Spectra of the forward stimulated Raman scattering (upper trace) and the backward stimulated Rayleigh-Kerr scattering (lower trace) in a $10-\mathrm{cm}$ long $\mathrm{CS}_{2}$ liquid cell pumped with 532-nm, 10-ns laser pulses of $1-\mathrm{cm}^{\prime \prime 1}$ linewidth at three different intensity levels. (See the explanation for Fig. 8.40 as well as reference [138] in Chapter 8.) 
(a)

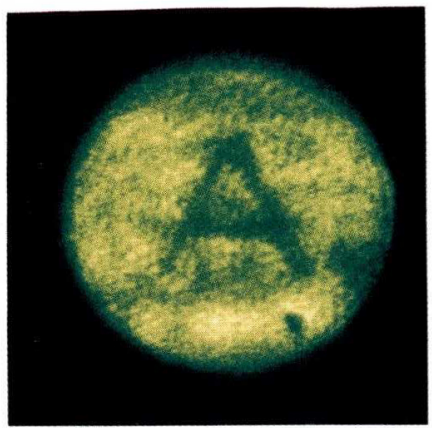

(c)

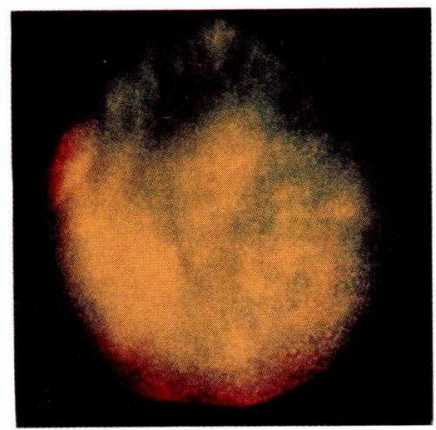

(e)

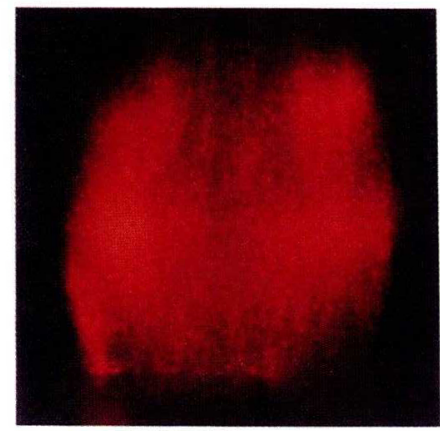

(b)

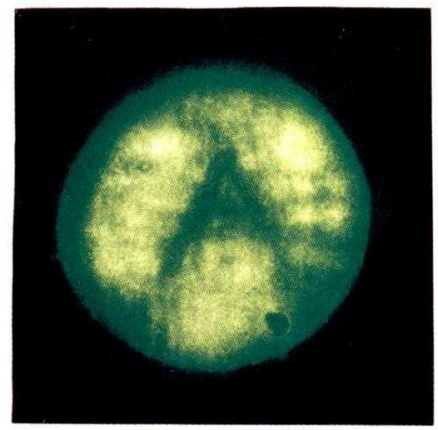

(d)

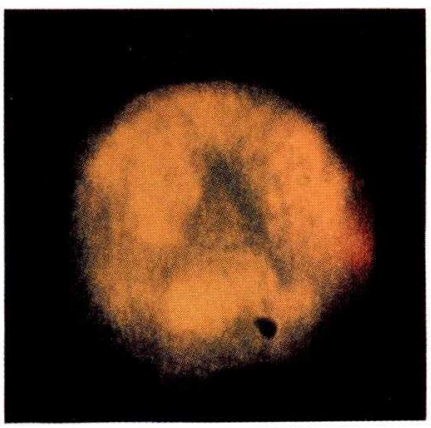

(f)

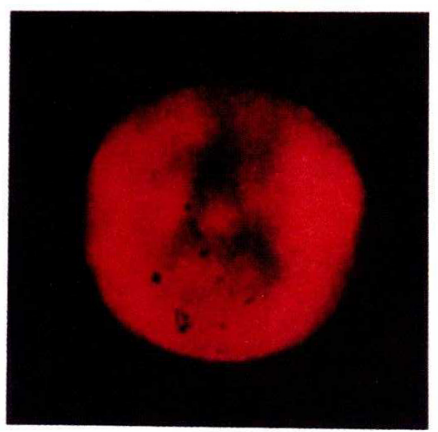

Photo 8 Experimental demonstration of optical phase-conjugation for backward stimulated scattering and emission. Near-field images carried by (a) the 532-nm (or 1064-nm) pump laser beam, (b) the backward stimulated Brillouin scattering from an acetone-filled vial, (c) and (d) the forward and backward two-photon pumped lasing from a dye-solution filled vial, (e) and (f) the forward and backward stimulated Raman scattering from a benzene-filled vial. (See the explanation for Fig. 9.28 in the text.) 


\section{Preface}

Nonlinear optics is a revolutionary extension of conventional (linear) optics promoted by laser technology. The main subject of nonlinear optics is the study of various effects and phenomena related to the interaction of intense coherent light with matter. A number of books on this topic have been published in the past. Some of them emphasize theoretical descriptions and mathematical formulations, whereas others emphasize technical aspects or experimental results.

This book is written based on the progress and achievements of nonlinear optics over the past forty-years. Within the same time-period, the authors have conducted fundamental research in the field of quantum electronics and published several books on nonlinear optics, laser physics, and laser devices.

This book focuses on both theoretical and experimental descriptions of various nonlinear optical effects and phenomena. Specifically, each chapter or each major section is composed of the following four basic parts: (1) Conceptual discussions and explanations of mechanisms; (2) Rigorous and concise mathematical formulation; (3) Typical experimental behavior and techniques; and (4) Up-to-date research achievements and applications.

In nonlinear optics and related areas, there are a tremendous number of published papers and conference presentations from the past four decades. However, only a very limited number of papers are cited in this book, based on the following three criteria: (i) original papers with the significance of novelty or innovation, (ii) earlier studies establishing basic understanding of the effects or phenomena, (iii) selected examples from a large number of similar studies. Although it is our desire to include all related papers of importance, due to the scope and size of this book, some of them are not cited. We would like to apologize for this.

We are deeply grateful to Prof. Yiping Cui (Southeast University, China), who has written Chapter 6 of this book and suggested the title of the book.

The authors wish to acknowledge those colleagues who have read the manuscript of the book and given their comments and suggestions: Prof. Hsiung Hsu (Ohio State University), Prof. Pao-Lo Liu (State University of New York at Buffalo), Dr. Yuzhen Shen, Dr. Liangmin Zhang, Dr. Yan Jiang, Mr. John B. Perry, and Mr. Ning Cheng.

We are indebted to those authors whose works have been quoted in this book. We are also grateful to many authors and publishers for the permission of reproducing their figures in this book.

Finally, one of us (G. S. H.) wishes to express special thanks to Dr. Paras N. Prasad, the Distinguished Professor and Director of Photonics Research Laboratory, State University of New York at Buffalo, for his valuable support and encouragement in conducting research activities. 
xiv

The International System of Units (SI) is used throughout this book for all formulations.

Photonics Research Laboratory

State University of New York at Buffalo

Buffalo, USA

Institute of Quantum Electronics

Song-Hao Liu

South China Normal University

Guangzhou, China

Guang-Sheng He

1999 


\section{CONTENTS}

1. Introduction to Nonlinear Optics 1

1.1 Definition of Nonlinear Optics 1

1.2 Major Events in History of Nonlinear Optics 5

1.3 Features of Interaction of Intense Light with Matter 8

1.4 Theoretical Framework of Nonlinear Optics 12

References 14

2. Fundamental Knowledge of Nonlinear Polarization 18

2.1 General Description of Optical Field-Induced Electric Polarization in Media $\quad 18$

2.2 Various Mechanisms Causing Nonlinear Polarization in a Medium $\quad 22$

2.3 Manipulation of Nonlinear Susceptibilities 24

2.4 Basic Properties of Nonlinear Susceptibilities 26

2.5 Nonlinear Coupled-Wave Equations 29

References 33

3. Second-Order Nonlinear Wave Mixing 35

3.1 Second-Harmonic Generation (SHG) 35

3.1.1 Basic Theoretical Descriptions 35

3.1.2 Mechanism Description by Quantum Theory of Radiation 40

3.1.3 Nonlinear Crystals for SHG 42

3.1.4 SHG Devices 46

3.2 Optical Sum- and Difference-Frequency Generation 47

3.2.1 Optical Sum-Frequency Generation 47

3.2.2 Optical Difference-Frequency Generation $\quad 49$

3.3 Optical Parametric Amplification and Oscillation $\quad 50$

3.3.1 General Description 50

3.3.2 Solutions of Coupled-Wave Equations $\quad 52$

3.3.3 Experimental Devices $\quad 55$

3.4 Special Second-Harmonic Generation $\quad 59$

3.4.1 Other Materials for SHG 59

3.4.2 SHG from Surfaces and Interfaces 59

References $\quad 61$ 
4. Third-Order Nonlinear Wave Mixing 63

4.1 Various Four-Wave Mixing (Four-Photon Parametric Interaction) Effects 63

4.2 Third-Harmonic Generation (THG) 66

4.2.1 Basic Theoretical Descriptions 66

4.2.2 Method of Phase-Matching for THG 69

4.2.3 Resonance Enhancement of THG 71

4.2.4 Materials and Devices for THG and Third-Order

Sum-Frequency Generation 73

4.3 Raman-Enhanced Four-Wave Mixing 77

4.3.1 Coherent Stokes- and Anti-Stokes Ring Emission 77

4.3.2 Raman-Enhanced Four-Wave Mixing with Two Incident Beams Having a Small Crossing Angle 80

4.4 Special Nonresonant Four-Photon Parametric Effects 81

4.4.1 Continuum Generation by Four-Photon Parametric Interaction 81

4.4.2 Frequency-Degenerate Four-Photon Parametric Interaction 84

4.5 Second-Harmonic Generation (SHG) by Third-Order Nonlinear Processes $\quad 86$

4.5.1 Electric Field-Induced SHG $\quad 86$

4.5.2 SHG in Optical Fibers $\quad 87$

$\begin{array}{ll}\text { References } & 89\end{array}$

5. Intense Light Induced Refractive-Index Changes 91

5.1 Description of Refractive Index in Linear Optics 91

5.2 Description of Refractive Index in Nonlinear Optics 94

5.3 Two-Wave Induced Refractive-Index Changes 96

5.3.1 Nonresonant Two-Wave Interaction 96

5.3.2 Two-Photon Resonance-Enhanced Two-Wave Interaction 98

5.3.3 Raman Resonance-Enhanced Two-Wave Interaction $\quad 100$

5.4 Mechanisms of Induced Refractive-Index Changes 102

5.4.1 Various Mechanisms of Induced Refractive-Index Changes 102

5.4.2 Molecular Reorientation Contribution 103

5.4.3 Optical Electrostriction Contribution 106

5.4.4 Temporal Behavior of Induced Refractive-Index Changes 107

5.5 Second-Order Nonlinearity Induced Refractive-Index

Change (Optical Pockels Effect) 110

References 115 
6. Photorefractive Effects 116

6.1 Principles and Physical Descriptions of Photorefractive Effects 117

$\begin{array}{ll}\text { 6.1.1 Rate Equations } & 117\end{array}$

6.1.2 Diffusion Field and Saturation Field $\quad 120$

6.1.3 Space Charge Field 121

6.2 Optical Wave Mixing in Photorefractive Media 125

6.2.1 Two-Wave Mixing $\quad 126$

6.2.2 Four-Wave Mixing 131

6.2.3 Beam Fanning 134

6.3 Photorefractive Materials and Properties 134

6.3.1 Inorganic Crystals 134

6.3.2 Organic and Polymeric Materials 136

References

138

7. Self-Focusing, Self-Phase-Modulation, and Spectral Self-Broadening

$\begin{array}{ll}\text { 7.1 Steady-State Self-Focusing Effect } & 140\end{array}$

$\begin{array}{ll}\text { 7.1.1 General Descriptions } & 140\end{array}$

7.1.2 Theory of Steady-State Self-Focusing 143

7.1.3 Another Formula for Steady-State Self-Focusing 149

7.2 Dynamic Self-Focusing Effect 149

7.2.1 Nonlinear Wave Equation for Dynamic Process 149

7.2.2 Features of Moving Foci 151

7.3 Self-Phase-Modulation and Spectral Self-Broadening 153

7.3.1 Self-Phase-Modulation and Frequency Chirp of Intense Short Light Pulses 153

7.3.2 Spectral Self-Broadening of Intense Short Light Pulses 157

7.3.3 Beat-Frequency Enhanced Spectral Self-Broadening $\quad 159$

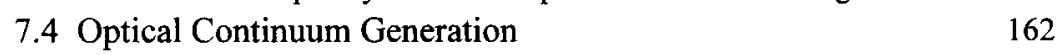

7.4.1 Continuum Generation with Ultrashort Laser Pulses 162

7.4.2 Continuum Generation with Nanosecond Laser Pulses $\quad 169$

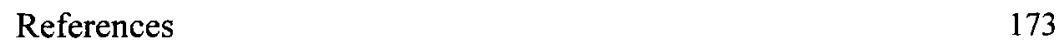

8. Stimulated Scattering of Intense Light 175

8.1 Introduction to Light Scattering 175

8.1.1 Origins of Light Scattering 175

8.1.2 Classification of Light Scattering 176

8.1.3 Differences between Spontaneous and Stimulated Scattering 179

8.1.4 Some Important Facts Related to Stimulated Scattering $\quad 181$ 
8.2 Theory of Stimulated Raman Scattering 182

8.2.1 Quantum-Electrodynamical Description of Raman Scattering 182

8.2.2 Probabilities of Spontaneous and Stimulated Raman Scattering 187

8.2.3 Gain Coefficient and Threshold Condition 189

8.2.4 Gain Coefficient Derived from Nonlinear Polarization Theory 193

8.2.5 Comparison of Two Theoretical Approaches 195

8.3 Experimental Studies of Stimulated Raman Scattering (SRS) 197

8.3.1 Raman Media and Experimental Setup 197

8.3.2 General Properties of SRS 199

8.3.3 Four-Wave Frequency Mixing (FWFM) in SRS Processes 201

8.3.4 Self-Focusing Effect in SRS Processes 210

8.3.5 Spin-Flip, Electronic, and Rotational SRS Effects 216

8.4 Stimulated Brillouin Scattering 224

8.4.1 Fundamental Description of Brillouin Scattering 224

8.4.2 Gain Coefficient of Stimulated Brillouin Scattering 227

8.4.3 Experimental Studies of Stimulated Brillouin Scattering (SBS) 235

8.5 Stimulated Rayleigh-Wing Scattering 239

8.6 Stimulated Kerr Scattering 244

8.6.1 Discovery of Super-Broadband Stimulated Scattering 244

8.6.2 Physical Model of Rayleigh-Kerr and Raman-Kerr Scattering 245

8.6.3 Cross Section of Kerr Scattering 247

8.6.4 Exponential Gain of Stimulated Kerr Scattering 252

8.6.5 Experimental Studies of Forward Stimulated Kerr Scattering 254

8.6.6 Experimental Studies of Backward Stimulated Kerr Scattering 260

References 265

9. Optical Phase-Conjugation 270

9.1 Introduction to Optical Phase Conjugation 270

9.1.1 Mathematical Definition of Optical Phase Conjugation Waves 270

9.1.2 Features of Optical Phase Conjugation Waves 272

9.2 Phase-Conjugation Wave (PCW) Generation via Four-Wave Mixing (FWM) 276

9.2.1 Theory of PCW Generation via Degenerate FWM 276

9.2.2 Holographic Model of PCW Generation via Degenerate FWM 279

9.2.3 Experimental Studies of PCW Generation via FWM 284

9.3 PCW Generation via Backward Stimulated Scattering 293

9.3.1 Phase-Conjugation Properties of Backward Stimulated Scattering 293

9.3.2 Theoretical Explanations: Quasi-Collinear and Partially Degenerate FWM Model 298

9.3.3 Theoretical Treatment in Unfocused-Beam Approximation $\quad 300$ 
9.4 PCW Generation via Backward Stimulated Emission

9.4.1 Single Pass Stimulated Emission from a Two-Photon Pumped (TPP) Lasing Medium

9.4.2 Phase-Conjugation Properties of the TPP Backward Stimulated Emission

9.4.3 Explanations of Phase-Conjugation Nature of Backward Stimulated Emission

9.4.4 Theoretical Treatment in Focused-Beam Approximation

9.4.5 Phase-Conjugation Properties of Backward Stimulated Emission from One-Photon Pumped Lasing Medium

10. Optical Coherent Transient Effects

10.1 Coherent Transient Interaction of Intense Light with Resonant Medium

10.2 Self-Induced Transparency 336

10.3 Photon Echo Effect 345

10.4 Optical Nutation Effect 354

10.4.1 Conceptual Description $\quad 354$

10.4.2 Optical Bloch Equation 356

10.4.3 Solution for Optical Nutation Effect 359

10.4.4 Experimental Studies $\quad 362$

10.5 Optical Free Induction Decay Effect 364

References $\quad 368$

11. Nonlinear Spectroscopic Effects 372

11.1 Major Spectral Broadening Mechanisms 372

11.2 Saturation Spectroscopy 377

11.2.1 General Descriptions of Saturated-Absorption Effect 377

11.2.2 Basic Theoretical Consideration 380

11.2.3 Experimental Setup and Results 382

11.2.4 Crossover Resonances in Saturation Spectroscopy $\quad 386$

11.3 Two-Photon Absorption Spectroscopy 388

11.3.1 General Description 388

11.3.2 Theoretical Considerations of TPA 390

11.3.3 Experiments 393

11.4 Coherent Raman Spectroscopy 396

11.4.1 General Descriptions 396

11.4.2 Coherent Anti-Stokes Raman Spectroscopy (CARS) 397

11.4.3 Raman-Induced Kerr Effect Spectroscopy (RIKES) 403

11.4.4 Raman Gain Spectroscopy (RGS) and Inverse Raman 
Spectroscopy (IRS) $\quad 405$

11.5 Laser Polarization Spectroscopy 408

11.5.1 Doppler-Free Saturated-Absorption Polarization

11.5.2 CARS Polarization Spectroscopy 411

11.5.3 Polarization Labeling Molecular Spectroscopy 413

$\begin{array}{ll}11.6 \text { Optogalvanic Spectroscopy } & 415\end{array}$

11.6.1 General Descriptions $\quad 415$

11.6.2 Experimental Setup $\quad 416$

11.6.3 Some Experimental Results $\quad 417$

$\begin{array}{ll}\text { References } & 420\end{array}$

12. Optical Bistability $\quad 422$

12.1 Basic Consideration of a Nonlinear F-P Etalon 422

12.1.1 Historical Background of Optical Bistability Studies $\quad 422$

12.1.2 Theory of Steady-State Optical Bistability 423

12.1.3 Dynamic Response of a Nonlinear F-P Etalon 427

12.2 General Knowledge of Optical Bistability Experiments 429

12.2.1 Influences of Spatial and Spectral Structures of the Incident Laser Beam on the Bistable Behavior 429

12.2.2 Standard Setup for Experimental Studies of Optical Bistability 431

12.2.3 The Earliest Observations of Optical Bistable Effects 432

12.3 Typical Performances of Various Optical Bistable Devices 435

12.3.1 Nonlinear Materials for Optical Bistable Studies $\quad 435$

12.3.2 Semiconductor Bistable Devices 436

12.3.3 Optical Waveguide Bistable Devices $\quad 439$

12.3.4 Transient Optical Bistable Behavior $\quad 441$

12.3.5 Hybrid Optical Bistable Devices 444

$\begin{array}{ll}\text { References } & 446\end{array}$

13. Optical Limiting, Stabilization, and Reshaping 448

13.1 Various Mechanisms for Optical Limiting 449

13.2 Opto-Thermal Effect-Based Optical Limiting 453

13.3 Reverse Saturable Absorption-Based Optical Limiting 457

13.4 Two-Photon Absorption-Based Optical Limiting 462

13.5 TPA-Based Optical Stabilization and Reshaping 468

$\begin{array}{ll}\text { References } & 475\end{array}$

14. Vigorous Theory of Nonlinear Susceptibilities 477

14.1 Density Matrix and Interaction Energy 477 
14.1.1 Basic Equations of Density Matrix $\quad 477$

14.1.2 Expression of Interaction Energy 479

14.2 Expressions of Susceptibilities Based on Density Matrix Solutions 483

14.2.1 Solutions of Density Matrix Equations 483

14.2.2 Explicit Formulations of Various-Order Susceptibilities 486

14.3 Properties of Nonlinear Susceptibilities 491

14.3.1 Local-Field Corrections $\quad 491$

14.3.2 Spatial Symmetry 494

14.3.3 Permutation Symmetry and Time-Reversal Symmetry of Susceptibilities 497

14.4 Resonant Enhancement of Nonlinear Susceptibilities $\quad 500$

$\begin{array}{ll}\text { 14.4.1 Introduction } & 500\end{array}$

14.4.2 Resonance-Enhancement of the First- and Second-Order Susceptibilities 501

14.4.3 Resonance-Enhancement of the Third-Order Susceptibility 502

14.5 Born-Oppenheimer Approximation of Nonlinear Susceptibilities $\quad 506$

14.5.1 Validity of Quantum-Mechanical Expressions of Nonlinear Susceptibility $\quad 506$

14.5.2 Feature of Born-Oppenheimer (B-O) Approximation $\quad 507$

14.5.3 General Expressions of Various-Order Polarization Components 510

14.5.4 Various-Order Susceptibilities in B-O Approximation 512 References

1. Physical Constants Commonly Used in Nonlinear Optics 519

2. Numerical Estimates and Conversion of Units 520

3. Tensor-Elements of the Linear Susceptibility for Crystals and other Media 523

4. Tensor-Elements of the Second-Order Susceptibility for Various Crystal Classes $\quad 524$

5. Tensor-Elements of the Susceptibility for Second-Harmonic Generation for Various Crystal Classes

6. Tensor-Elements of the Third-Order Susceptibility for Crystals and other Media

7. Tensor-Elements of the Nuclear Third-Order Susceptibility in Born-Oppenheimer Approximation 
xxii

8. The Solution of Eq. (9.4-15) 537

9. Derivation of Formulae for Self-Induced Transparency of $2 \pi$-Pulse

Index 